\title{
Recognition of Paths and Curves in the 2-Dimensional Euclidean Geometry
}

\author{
İdris Ören* and Djavvat Khadjiev \\ (Communicated by Levent Kula)
}

\begin{abstract}
This paper presents global differential invariants of curves and paths in the 2-dimensional Euclidean geometry for the groups of Euclidean transformations $M(2)$ and special Euclidean transformations $M^{+}(2)$. For these groups, analogues of the fundamental theorem for Euclidean curves are obtained in terms of global differential invariants of a path and a curve. Moreover, for given two paths(or curves) with the common differential $G$-invariants, evident forms of all Euclidean transformations that maps one of the paths (or curves) to the other are found.
\end{abstract}

Keywords: Path; invariant; Euclidean geometry.

AMS Subject Classification (2020): Primary: 53A04; Secondary: 53A55.

\section{Introduction}

Let $E_{n}$ be the $n$-dimensional Euclidean space. The groups of all orthogonal and all special orthogonal transformations of $E_{n}$ is denoted by $O(n)$ and $O^{+}(n)$, resp. Let $M(n)$ be group of all Euclidean transformations of $E_{n}$ and $M^{+}(n)$ be group of all special Euclidean transformations of $E_{n}$.

In the classical theory of curves in $E_{n}$, where $n \geq 3$, using Frenet-Serret equations, curvature functions $k_{i}, i=$ $1, \ldots, n-1$, of the curve were obtained (see [1, p.172]). The function $k_{i}$ is $M(n)$-invariant for $i=1, \ldots n-2$. But $k_{n-1}$ is not $M(n)$-invariant. It is $M^{+}(n)$-invariant. In the case $n=2$, two different concepts of curvatures were defined: the signed curvature $\kappa_{ \pm}=\frac{\left[x^{\prime}(t) x^{\prime \prime}(t)\right]}{\left\langle x^{\prime}(t), x^{\prime}(t)\right\rangle^{\frac{3}{2}}}$ (see [4, p.64-66], [5, p.14-15],[6, p.25], [19, p.8]) and the curvature $\kappa(x)=\frac{\left|\left[x^{\prime}(t) x^{\prime \prime}(t)\right]\right|}{\left\langle x^{\prime}(t), x^{\prime}(t)>^{\frac{3}{2}}\right.}$ (see [1, p.31], [20, p.21]). The function $\kappa_{ \pm}$is $M^{+}(2)$-invariant, but it is not $M(2)$ invariant. The function $\kappa$ is $M(2)$-invariant. The signed curvature $\kappa_{ \pm}$is more used for investigation of curves in two dimensional classical differential geometry (see [4, p,64-66], [5, p.14-15]). Thus invariant theory of curves in the classical differential geometry was developed only for the group $M^{+}(n)$. In addition, the method of orthogonal frame in the classical differential geometry give conditions only for the local $M^{+}(n)$-equivalence of curves (see [16, p,9-19]).

In works [2, 7], by using invariant parametrization of curves, the problem of $G$-equivalence of curves (that is nonparametric curves) was reduced to the problem of $G$-equivalence of paths (that is parametric curves) for $G=M(n), M^{+}(n)$. Complete systems of global $G$-invariants of regular paths and regular curves in classical geometries were obtained in $[2,7]$. This approach was developed for curves in papers $[10,11,17,18]$ and for vector fields in $[8,9]$.

In books ([4, Theorems 6.1 and 6.8], [5, p.136-137]) existence and uniqueness theorems for regular parametric curves (that is paths) in $E_{2}$ were obtained for the group $G=M^{+}(2)$.

The present paper is devoted to an investigation of problems of $G$-equivalence of paths and curves in $E_{2}$ for groups $G=M(2)$ and $G=M^{+}(2)$ in terms of global $G$-invariants of paths and curves. We give complete 
systems of global $G$-invariants of regular paths and regular curves. Existence and uniqueness theorems are obtained.

Let $G=M(2)$ or $G=M^{+}(2)$. The following is the structure of the present paper. In Sect.2, Euclidean motions groups are redefined using complex numbers and its algebraic properties. In Sect. 3, descriptions of invariant parametrizations of curves and the theorem on reduction are given. In Sec.4, definitions of all types of paths are introduced. The global equivalence conditions of paths are found for the groups $G$. The existence and uniqueness theorems of paths and curves are given. In Sec. 5, for given two paths with the common differential $G$-invariants, evident forms of all Euclidean transformations that maps one of the paths to the other are given. In Sect. 6, existence of rigidity theorems for regular curves are given. In Sect. 7, we obtain the global equivalence conditions of regular curves for the group $M(2)$.

\section{Preliminaries}

Let $\mathbb{C}$ be the field of complex numbers. Any complex number $u \in \mathbb{C}$ can be written in the form $u=u_{1}+i u_{2}$, where $u_{1}, u_{2} \in \mathbb{R}, i^{2}=-1$. It follows that the multiplication of two complex numbers $u$ and $v$, denoted by $u v$, is defined by

$$
u v=\left(u_{1}+i u_{2}\right)\left(v_{1}+i v_{2}\right)=\left(u_{1} v_{1}-u_{2} v_{2}\right)+i\left(u_{1} v_{2}+u_{2} v_{1}\right)
$$

Consider the complex number $u=u_{1}+i u_{2}$ in the matrix form $u=\left(\begin{array}{l}u_{1} \\ u_{2}\end{array}\right)$. Then, the equality above $u v$ can be rewritten as

$$
u v=\left(\begin{array}{l}
u_{1} v_{1}-u_{2} v_{2} \\
u_{1} v_{2}+u_{2} v_{1}
\end{array}\right)=\left(\begin{array}{cc}
u_{1} & -u_{2} \\
u_{2} & u_{1}
\end{array}\right)\left(\begin{array}{l}
v_{1} \\
v_{2}
\end{array}\right)
$$

Denote by $L_{u}$ the matrix $\left(\begin{array}{cc}u_{1} & -u_{2} \\ u_{2} & u_{1}\end{array}\right)$. Then the equality (2.1) can be written in the following form

$$
u v=L_{u} v, \quad \forall u, v \in \mathbb{C}
$$

The field $\mathbb{C}$ can be used to represents $E_{2}$ with the inner product $\langle u, v\rangle=u_{1} v_{1}+u_{2} v_{2}$ for all $u=u_{1}+i u_{2}, v=$ $v_{1}+i v_{2} \in \mathbb{C}$. Here, the quadratic form on $E_{2}$ is $\langle u, u\rangle$ for all $u \in \mathbb{C}$. The conjugate of $u$, denoted by $\bar{u}$, is defined as $\bar{u}=u_{1}-i u_{2}$. Then we have $u+\bar{u}=2 u_{1}$ and $\langle\bar{u}, \bar{v}\rangle=\langle u, v\rangle$. For $u \neq 0$, the inverse of $u$ is defined as $\frac{1}{u}=\frac{\bar{u}}{<u, u>}$. Moreover, let $\Lambda=\left(\begin{array}{cc}1 & 0 \\ 0 & -1\end{array}\right)$. Then we have $\bar{u}=\Lambda u$.

Put $\mathbb{C}^{*}=\{u \in \mathbb{C} \mid u \neq 0\}$. For $u=u_{1}+i u_{2} \in \mathbb{C}^{*}$, we have $P_{u}=\left(\begin{array}{cc}\frac{u_{1}}{\sqrt{<u, u>}} & \frac{-u_{2}}{\sqrt{<u, u\rangle}} \\ \frac{u_{2}}{\sqrt{\langle u, u\rangle}} & \frac{u_{1}}{\sqrt{\langle u, u\rangle}}\end{array}\right)$ and $L_{u}=\sqrt{\left\langle u, u>P_{u}\right.}$ such that $P_{u} \in O^{+}(2)$.

Let $\left(\begin{array}{ll}u_{1} & v_{1} \\ u_{2} & v_{2}\end{array}\right)$ be a matrix corresponding to complex numbers $u=u_{1}+i u_{2}, v=v_{1}+i v_{2}$ and its determinant will be denoted by $[u v]$.

Proposition 2.1. Let $u, v \in \mathbb{C}$ be two complex numbers such that $u \neq 0$. Then the complex number $v u^{-1}$ exists and the number has the form

$$
v u^{-1}=\frac{v}{u}=\frac{<u, v>}{<u, u>}+i \frac{[u v]}{<u, u>}
$$

and

$$
L_{\frac{v}{u}}=\left(\begin{array}{cc}
\frac{\langle u, v\rangle}{<u, u>} & -\frac{[u v]}{<u, u>} \\
\frac{[u v]}{<u, u>} & <u, v> \\
\langle u, u>
\end{array}\right) .
$$

Proof. The proof is known from [12, Proposition 14]. 
Put $S\left(\mathbb{C}^{*}\right)=\{u \in \mathbb{C}|| u \mid=1\}, P\left(\mathbb{C}^{*}\right)=\left\{L_{u} \mid u \in \mathbb{C}^{*}\right\}$ and $P\left(S\left(\mathbb{C}^{*}\right)=\left\{L_{u} \mid u \in S\left(\mathbb{C}^{*}\right)\right\} . S\left(\mathbb{C}^{*}\right)\right.$ is a subgroup of the group $\mathbb{C}^{*}$. Denote the set of all matrices $\left\{g W \mid g \in P\left(\mathbb{C}^{*}\right)\right\}$ by $P\left(\mathbb{C}^{*}\right) W$, where $g W$ is the multiplication of matrices $g$ and $W$.

Put $O^{-}(2)=\left\{g W: g \in O^{+}(2)\right\}$. Then $O^{-}(2) \subset O(2), O^{+}(2) \cap O^{-}(2)=\emptyset$ and the set $O^{+}(2)$ is a subgroup of $O(2)$.

The following theorem is known from [3, p.172].

Theorem 2.1. $\quad$ (i) $O^{+}(2)=\left\{F: E_{2} \rightarrow E_{2} \mid F(x)=L_{u} x, u \in S\left(\mathbb{C}^{*}\right), \forall x \in E_{2}\right\}$

(ii) $O^{-}(2)=\left\{F: E_{2} \rightarrow E_{2} \mid F(x)=L_{u} W(x), u \in S\left(\mathbb{C}^{*}\right), \forall x \in E_{2}\right\}$

(iii) $O(2)=O^{+}(2) \cup O^{-}(2)$.

(iv) $M^{+}(2)=\left\{F: E_{2} \rightarrow E_{2} \mid F(x)=L_{u} x+b, u \in S\left(\mathbb{C}^{*}\right), b \in E_{2}, \forall x \in E_{2}\right\}$

(v) $M^{-}(2)=\left\{F: E_{2} \rightarrow E_{2} \mid F(x)=L_{u} W(x)+b, u \in S\left(\mathbb{C}^{*}\right), b \in E_{2}, \forall x \in E_{2}\right\}$

(vi) $M(2)=M^{+}(2) \cup M^{-}(2)$.

\section{Descriptions of invariant parametrizations of curves and the theorem on reduction}

Let $I=(a, b) \subseteq \mathbb{R}$ and

$$
\xi: I \rightarrow E_{2}
$$

be a $C^{2}$-mapping and we denote by $\xi(t)=\left(\xi_{1}(t), \xi_{2}(t)\right)$. Clearly the path $\xi$ is called an $I$-path in $E_{2}$. The functions $\xi_{1}(t)$ and $\xi_{2}(t)$ are real $C^{2}$-functions on $I$. For convenience, we prefer $\xi$ instead of $\xi(t)$.

Let $I_{1}=(a, b)$ and $I_{2}=(c, d)$ be two intervals in $\mathbb{R}$.

Definition 3.1. (see [2]) Let $\xi\left(t_{1}\right)$ be an $I_{1}$-path and be $\eta\left(t_{2}\right)$ an $I_{2}$-path in $E_{2}$. If a $C^{(2)}$-diffeomorphism $\psi: I_{2} \rightarrow I_{1}$ exists such that $\psi^{\prime}\left(t_{2}\right)>0$ and $\eta\left(t_{2}\right)=\xi\left(\psi\left(t_{2}\right)\right), \forall t_{2} \in I_{2}$, we say that the paths $\xi\left(t_{1}\right)$ and $\eta\left(t_{2}\right)$ are $D$-equivalent. A class of $D$-equivalent paths in $E_{2}$ will be called a curve (non-parametrized curve) in $E_{2}$ and denote it by $\Phi$. Moreover, we say that a path $\xi \in \Phi$ is called a parametrization of a curve $\Phi$.

Let $G=M(2)$ or $G=M^{+}(2)$.

Definition 3.2. Let two paths $\xi$ and $\eta$ be defined on the same interval $I$. Then these paths are called $G$ equivalent if there is $F \in G$ such that $\eta(t)=F \xi(t)$ for all $t \in I$. In this case, it will be denoted by $\xi(t) \stackrel{G}{\sim} \eta(t)$.

Let $\Phi=\left\{\nu_{\tau}, \tau \in \Pi\right\}$ be a curve, where $\nu_{\tau}$ is a parametrization of $\Phi$. Then $F \Phi=\left\{F \nu_{\tau}, \tau \in \Pi\right\}$ is a curve for all $F$ in $G$.

Definition 3.3. Two curves $\Phi$ and $\Psi$ are called $G$-equivalent if there is some $F \in G$ such that $\Psi=F \Phi$. In this case, it will be denoted by $\Phi \stackrel{G}{\sim} \Psi$.

Let $\zeta_{1}, \zeta_{2}, \ldots, \zeta_{n}: I \rightarrow E_{2}$ are paths defined on $I$.

Definition 3.4. A function $\psi\left(\zeta_{1}, \zeta_{2}, \ldots, \zeta_{n}\right)$ is called $G$-invariant if $\psi\left(F \zeta_{1}, F \zeta_{2}, \ldots, F \zeta_{n}\right)=\psi\left(\zeta_{1}, \zeta_{2}, \ldots, \zeta_{n}\right) \mathrm{i} \forall F \in$ $G$ and $\forall t \in I$.

For $\xi^{\prime}, \xi^{\prime \prime}$ of $\xi$, the determinant of the matrix $\left(\begin{array}{ll}\xi_{1}^{\prime} & \xi_{1}^{\prime \prime} \\ \xi_{2}^{\prime} & \xi_{2}^{\prime \prime}\end{array}\right)$ will be denoted by $\left[\xi^{\prime} \xi^{\prime \prime}\right]$.

Example 3.1. Let $\xi(t), \eta(t)$ be $I$-paths in $E_{2}$. By the definitions of the group $O(2)$, the bilinear form $\langle\xi(t), \eta(t)>$ is $O(2)$-invariant. This implies that the bilinear form $<\xi^{\prime}(t), \eta^{\prime}(t)>$ is $M(2)$-invariant. 
Example 3.2. Let $\xi(t)=\left(\begin{array}{c}\xi_{1}(t) \\ \xi_{2}(t)\end{array}\right), y(t)=\left(\begin{array}{c}\eta_{1}(t) \\ \eta_{2}(t)\end{array}\right)$ be $I$-paths in $E_{2}$. Denote the determinant $\mid \begin{array}{ll}\xi_{1}(t) & \eta_{1}(t) \\ \xi_{2}(t) & \eta_{2}(t)\end{array}$ by $[\xi(t) \eta(t)]$. Since $\operatorname{det}(g)=1$ for all $g \in O^{+}(2)$, we have $[(g \xi(t))(g \eta(t))]=\operatorname{det}(g)[\xi(t) \eta(t)]=[\xi(t) \eta(t)]$ for all $g \in O^{+}(2)$. Hence $[\xi(t) \eta(t)]$ is $O^{+}(2)$-invariant function. This implies that the bilinear form $\left[\xi^{\prime}(t) \eta^{\prime}(t)\right]$ is $M^{+}(2)-$ invariant.

Example 3.3. The function $[\xi(t) \eta(t)]$ is $O^{+}(2)$-invariant. Using $\operatorname{det}(W)=-1$, we obtain $|[\bar{\xi}(t) \bar{\eta}(t)]|=$ $|[(W \xi(t))(W \eta(t))]|=|\operatorname{det}(W)[\xi(t) \eta(t)]|=|[\xi(t) \eta(t)]|$. Hence the function $|[\xi(t) \eta(t)]|$ is $O(2)$-invariant. This implies that the function $\left|\left[\xi^{\prime}(t) \eta^{\prime}(t)\right]\right|$ is $M(2)$-invariant. Similarly, the function $[\xi(t) y(t)]^{2}$ is $O(2)$-invariant. This implies that the function $\left[\xi^{\prime}(t) \eta^{\prime}(t)\right]^{2}$ is $M(2)$-invariant.

Definition 3.5. A $C^{(2)}$-mapping $\xi: I \rightarrow E_{2}$ is called regular $I$-path if $\xi^{\prime}(t) \neq 0$ for all $t \in I$.

For example, let $\xi(t)=\left(t, t^{2}\right)$ be an $\mathbb{R}$-path. Then, $\xi^{\prime}(t)=(1,2 t) \neq 0$ for all $t \in \mathbb{R}$. Hence, $\xi(t)$ is a regular $\mathbb{R}$ path.

A curve is called regular if it contains a regular path.

Proposition 3.1. Let $\Phi$ be a regular curve. Then every $\xi \in \Phi$ is a regular path.

Proof. It is clear from [12, Proposition 37].

The arc length of the regular $I$-path $\xi$ from $t=c$ to $t=d$ is defined as follows:

$\ell_{\xi}(c, d)=\int_{c}^{d} \frac{\left|\left[\xi^{\prime}(t) \xi^{\prime \prime}(t)\right]\right|}{<\xi^{\prime}(t), \xi^{\prime}(t)>} d t$, for $c, d \in I=(a, b) \subseteq \mathbb{R}$ and $c<d$. Then there are the $\operatorname{limits}_{c \rightarrow a} \ell_{\xi}(c, d) \leq+\infty$ and $\lim _{d \rightarrow b} \ell_{\xi}(c, d) \leq+\infty$. These limits denoted by $\ell_{\xi}(a, d)$ and $\ell_{\xi}(c, b)$, resp.

Assume the limits $\ell_{\xi}(a, d)=\lim _{c \rightarrow a} \ell_{\xi}(c, d) \leq+\infty$ and $\ell_{\xi}(c, b)=\lim _{d \rightarrow b} \ell_{\xi}(c, d) \leq+\infty$ exist. Then the following statements exist:

$\left(\delta_{1}\right) 0<\ell_{\xi}(a, d)<+\infty, \quad 0<\ell_{\xi}(c, b)<+\infty$.

$\left(\delta_{2}\right) 0<\ell_{\xi}(a, d)<+\infty, \quad \ell_{\xi}(c, b)=+\infty$.

$\left(\delta_{3}\right) \ell_{\xi}(a, d)=+\infty, \quad 0<\ell_{\xi}(c, b)<+\infty$.

$\left(\delta_{4}\right) \ell_{\xi}(a, d)=+\infty, \quad \ell_{\xi}(c, b)=+\infty$.

Assume that the case $\left(\delta_{1}\right)$ or $\left(\delta_{2}\right)$ satisfies for some $c, d \in I$. Then $l=\ell_{\xi}(a, d)+\ell_{\xi}(c, b)-\ell_{\xi}(c, d)$, where $0 \leq l \leq+\infty$.

According to the group $M(2)$, we introduce the type of a regular $I$-path $\xi$. The type of a regular path $\xi$ will be denoted by $L_{\xi}$. In the cases $\left(\delta_{1}\right)$ and $\left(\delta_{2}\right)$, the type of the regular path $\xi$ is $(0, l)$. In the cases $\left(\delta_{3}\right)$ and $\left(\delta_{4}\right)$, the types of the regular path $\xi$ are $(-\infty, 0)$ and $(-\infty,+\infty)$, resp. All types of the regular paths are $(0, l)$, where $l<+\infty,(0,+\infty),(-\infty, 0)$, and $(-\infty,+\infty)$.

The proofs of the following propositions are similar to proofs of propositions in [2].

Proposition 3.2. $\quad(i)$ Let $\xi$ and $\eta$ be $M(2)$-equivalent. Then $L_{\xi}=L_{\eta}$.

(ii) Let $\xi, \eta \in \Phi$. Then $L_{\xi}=L_{\eta}$.

According to the group $M(2)$, the type of a regular path $\xi \in \Phi$ is called the type of the curve $\Phi$ and denoted by $L_{\Phi}$.

Proposition 3.3. Let two curves $\Phi$ and $\Psi$ be $M(2)$-equivalent. Then $L_{\Phi}=L_{\Psi}$.

For all types of the group $M(2)$, the length of an arc $s_{\xi}(t)$ for a regular $I$-path $\xi$ is defined in accordance with its the type as follows:

In the case $L_{\xi}=(0, l)$, where $l \leq+\infty$, we define $s_{\xi}(t)=\ell_{\xi}(a, t)$. In the case $L_{\xi}=(-\infty, 0)$, we define $s_{\xi}(t)=$ $-\ell_{\xi}(t, b)$. In each interval $I=(a, b)$ of the line $\mathbb{R}$, we choose a fixed point and denote it by $x_{I}$. In the case $I=(-\infty,+\infty)$, we choose $x_{I}=0$. We put $s_{\xi}(t)=\ell_{\xi}\left(x_{I}, t\right)$ for the interval $I$.

Since $\xi$ is a regular path, $\frac{d s_{\xi}}{d t}>0$. Then an inverse function of the function $s_{\xi}(t)$ exists and denote it by $t_{\xi}(s)$. Clearly, the domain of definition of the function $t_{\xi}(s)$ is $L_{\xi}$.

We omit the easy proofs of the following Propositions 3.4, 3.5, 3.6, 3.7.(see [2]). 
Proposition 3.4. Let $I=\left(a_{1}, b_{1}\right)$ and $J=\left(a_{2}, b_{2}\right)$. For I-path $\xi$, the following statements hold:

(i) $s_{F \xi}(t)=s_{\xi}(t)$ and $t_{F \xi}(s)=t_{\xi}(s)$ for all $t \in I$, for all $s \in L_{\xi}$ and all $F \in M(2)$.

(ii) for any $C^{2}$-diffeomorphism with $\psi^{\prime}(r)>0$ for all $r \in I$, the following equalities hold: $s_{\xi(\psi)}(r)=s_{\xi}(\psi(r))+$ $a_{0}, \forall r \in I$, and $\psi\left(t_{\xi(\psi)}\left(s+a_{0}\right)\right)=t_{\xi}(s), \forall s \in L_{\xi}$. Here, $a_{0}=0$ for $L_{\xi} \neq(-\infty,+\infty)$ and $a_{0}=\ell_{\xi}\left(\psi\left(a_{J}\right), a_{I}\right)$ for $L_{\xi}=(-\infty,+\infty)$.

According to Proposition 3.4, we have $\xi\left(t_{\xi}(s)\right) \in \Phi$.

Definition 3.6. (see [2]) $\xi\left(t_{\xi}(s)\right) \in \Phi$ is called an invariant parametrization of $\Phi$.

Denote the set of all invariant parameterizations of a curve $\Phi$ by $P_{\Phi}$.

Proposition 3.5. Let $\xi \in \Phi$ and $\xi$ be a I-path, where $I=L_{\Phi}$. Then the followings are equivalent:

(i) $\xi \in \Phi$ is an invariant parametrization.

(ii) $\frac{\|\left[\xi^{\prime}(t) \xi^{\prime \prime}(t)\right] \mid}{<\xi^{\prime}(t), \xi^{\prime}(t)>}=1, \forall s \in L_{\Phi}$.

(iii) $s_{\xi}(s)=s, \forall s \in L_{\Phi}$.

Proof. A similar proof is given in [2, Proposition 3].

In the case $s_{\xi}(s)=s, \forall s \in L_{\Phi}, s$ is called an invariant parameter of $\Phi$.

Assume that $I$ is one of the intervals $(0, l), l<+\infty ;(0,+\infty),(-\infty, 0)$ or $(-\infty,+\infty)$.

Proposition 3.6. Let $L_{\Phi} \neq(-\infty,+\infty)$. Then a unique invariant parametrization of $\Phi$ exists.

Remark 3.1. For $L_{\Phi}=(-\infty,+\infty), P_{\Phi}$ is infinite and uncountable. Moreover, if $\xi(t)$ is a periodic path then $L_{\xi}=(-\infty,+\infty)$.

Proposition 3.7. Let $\xi \in P_{\Phi}$ and $L_{\Phi}=(-\infty,+\infty)$. Then

$P_{\Phi}=\{\eta: \eta(s)=\xi(s+u), u \in(-\infty,+\infty)\}$.

The following theorem is given in [2, Theorem 1] for $n$-dimensional Euclidean space.

Theorem 3.1. Let $\Phi$ and $\Psi$ are regular curves and $\xi \in P_{\Phi}, \eta \in P_{\Psi}$ are invariant parametrizations.

(i) In the case $L_{\Phi}=L_{\Psi} \neq(-\infty,+\infty)$, $\Phi$ and $\Psi$ are G-equivalent if and only if $\xi$ and $\eta$ are G-equivalent.

(ii) In the case $L_{\Phi}=L_{\Psi}=(-\infty,+\infty)$, $\Phi$ and $\Psi$ are $G$-equivalent if and only if $\xi$ and $\eta\left(\psi_{x}\right)$ are $G$-equivalent for some $x \in(-\infty,+\infty)$, where $\psi_{x}(s)=s+x$.

Theorem 3.1 reduces the problem of G-equivalence of curves to the problem of G-equivalence of paths in the case $L_{\Phi}=L_{\Psi} \neq(-\infty,+\infty)$. But we cannot claim the same in the case $L_{\Phi}=L_{\Psi}=(-\infty,+\infty)$.

Definition 3.7. $\mathbb{R}$-paths $\xi$ and $\eta$ are called $[G,(-\infty,+\infty)]$-equivalent if there exist $g \in G$ and $d \in \mathbb{R}$ such that $\eta=g \xi(t+d)$ for all $t \in \mathbb{R}$.

Let $\Phi$ and $\Psi$ be two curves, where $L_{\Phi}=L_{\Psi}=(-\infty,+\infty)$. Then, Theorem 3.1 reduces the $G$-equivalence of these curves to $[G,(-\infty,+\infty)]$-equivalent of paths.

\section{Equivalence of paths for the groups $M(2)$ and $M^{+}(2)$}

Proposition 4.1. Let $\xi$ and $\eta$ be two I-paths. Then,

(i) $\xi$ and $\eta$ are $M^{+}(2)$-equivalent if and only if $\xi^{\prime}$ and $\eta^{\prime}$ are $O^{+}(2)$-equivalent.

(ii) $\xi$ and $\eta$ are $M(2)$-equivalent if and only if $\xi^{\prime}$ and $\eta^{\prime}$ are $O(2)$-equivalent. 
Proof. (i) $\Rightarrow$ : Let $\xi$ and $\eta$ be $M^{+}(2)$-equivalent. Then, by Theorem 2.1, there is $F \in M^{+}(2)$ such that $\eta(t)=$ $F \xi(t)=P_{u} \xi(t)+b$ for the constant $b$ in $E_{2}$ and all $t \in I$. This equality implies $\eta^{\prime}=P_{u} \xi^{\prime}$. Then, we obtain that $\xi^{\prime}$ and $\eta^{\prime}$ are $O^{+}(2)$-equivalent.

$\Leftarrow$ : Let $\xi^{\prime}$ and $\eta^{\prime}$ be $O^{+}(2)$-equivalent. Then, by Theorem 2.1, there is $F \in O^{+}\left(2\right.$ such that $\eta^{\prime}(t)=(F \xi(t))^{\prime}$ for all $t \in I$. This equality implies that $\eta^{\prime}(t)-(F \xi(t))^{\prime}=(\eta(t)-F \xi(t))^{\prime}=0$ for all $t \in I$. Then there is a constant $b \in \mathbb{C}$ such that $\eta(t)=F \xi(t)+b$. That is, $\xi$ and $\eta$ are $M^{+}(2)$-equivalent.

(ii) The proof is clear from the case $(i)$.

Remark 4.1. Proposition 4.1 reduces the problem of $M(2)$-equivalence and $M^{+}(2)$-equivalence of $I$-paths to the $O(2)$-equivalence and $O^{+}(2)$-equivalence of $I$-paths, resp.

Let $\xi(t)$ be a regular $I$-path. For shortness, we put $f_{\xi}(t)=<\xi^{\prime}(t), \xi^{\prime}(t)>, \quad g_{\xi}(t)=\left[\xi^{\prime}(t) \xi^{\prime \prime}(t)\right], \quad h_{\xi}(t)=$ $\left[\xi^{\prime}(t) \xi^{\prime \prime}(t)\right]^{2}$ and $k_{\xi}(t)=<\xi^{\prime \prime}(t), \xi^{\prime \prime}(t)>$.

Proposition 4.2. $\quad$ (i) The functions $f_{\xi}(t), k_{\xi}(t)$ and $h_{\xi}(t)$ are $M(2)$-invariant.

(ii) The function $g_{\xi}(t)$ is $M^{+}(2)$-invariant.

Proof. It is clear from [12, Proposition 13] .

Theorem 4.1. Let $\xi$ and $\eta$ be two regular I-paths. Then

$$
\xi^{M^{+}(2)} \eta \Leftrightarrow\left\{\begin{array}{l}
f_{\xi}(t)=f_{\eta}(t) \\
g_{\xi}(t)=g_{\eta}(t)
\end{array}\right.
$$

for all $t \in I$.

Moreover, there exists the unique $F \in M^{+}(2)$ such that $\eta(t)=F \xi(t)=N \xi(t)+b$, where $N \in O^{+}(2)$ and $b \in E_{2}$ which are not depend on $t$ in $I$ have the forms

$$
N=\left(\begin{array}{cc}
\frac{\left\langle\xi^{\prime}(t), \eta^{\prime}(t)>\right.}{f_{\xi}(t)} & -\frac{\left[\xi^{\prime}(t) \eta^{\prime}(t)\right]}{f_{\xi}(t)} \\
\frac{\left[\xi^{\prime}(t) \eta^{\prime}(t)\right]}{f_{\xi}(t)} & \frac{\left\langle\xi^{\prime}(t), \eta^{\prime}(t)>\right.}{f_{\xi}(t)}
\end{array}\right)
$$

and

$$
b=\eta(t)-N \xi(t)
$$

$\forall t \in I$, resp.

Proof. A proof of this theorem is obtained from Proposition 4.1 and [15, Theorem 3.2].

Example 4.1. Let $\xi(t)=\left(t^{2}, e^{t}\right)$ and $\eta(t)=\left(\frac{3}{5} t^{2}-\frac{4}{5} e^{t}+1, \frac{4}{5} t^{2}+\frac{3}{5} e^{t}+2\right)$ be two $\mathbb{R}$-paths. These paths are regular and the equalities (4.1) hold. Then, by Theorem 4.1, $\xi(t)$ and $\eta(t)$ are $M^{+}(2)$-equivalent. Further, using Theorem 4.1 , we have $N=\left(\begin{array}{cc}\frac{3}{5} & \frac{-4}{5} \\ \frac{4}{5} & \frac{3}{5}\end{array}\right)$ and $b=1+2 i$.

Definition 4.1. (see [4, p.64-66], [5, p.14-15], [6, p.25], [19, p.8]) The function $\frac{g_{\xi}(t)}{f_{\xi}(t)^{\frac{3}{2}}}$ is called the signed curvature of a regular path $\xi(t)$ in $E_{2}$ and denoted by $\kappa_{ \pm}(\xi)$.

Theorem 4.2. Let $\xi$ and $\eta$ be two regular I-paths. Then

$$
\xi \stackrel{M^{+}(2)}{\sim} \eta \Leftrightarrow\left\{\begin{array}{c}
f_{\xi}(t)=f_{\eta}(t) \\
\kappa_{ \pm}(\xi)=\kappa_{ \pm}(\eta) .
\end{array}\right.
$$

for all $t \in I$.

Moreover, there exists the unique $F \in M^{+}(2)$ such that $\eta(t)=F \xi(t)=N \xi(t)+b$, where $N \in O^{+}(2)$ and $b \in E_{2}$ which are not depend on $t$ in I have the forms (4.2) and (4.3),resp. 
Proof. Assume that the paths $\xi(t)$ and $\eta(t)$ are $M^{+}(2)$-equivalent. Since functions $f_{\xi}(t)=<\xi^{\prime}(t), \xi^{\prime}(t)>$ and $\kappa_{ \pm}(\xi)$ are $M^{+}(2)$-invariant, equalities (4.4) hold.

Conversely, assume that (4.4) holds. The equality $\kappa_{ \pm}(\xi)=\frac{g_{\xi}(t)}{f_{\xi}(t)^{\frac{3}{2}}}$ implies $g_{\xi}(t)=\kappa_{ \pm}(\xi) f_{\xi}(t)^{\frac{3}{2}}$. This equality and (4.4) imply the equality $g_{\xi}(t)=g_{\eta}(t)$. Hence equalities (4.1) hold. Then, using Theorem 4.1 , we obtain the unique special Euclidean transformation $F$ of $E_{2}$ such that $\eta(t)=F \xi(t)=N \xi(t)+b$, where $N \in O^{+}(2)$ and the constant $b$ in $E_{2}$.

Remark 4.2. In [4, p.85-87,Lemma 6.6,Theorem 6.8] the part " (4.4) if and only if $\xi(t)$ and $\eta(t)$ are $M^{+}(2)$ equivalent " of Theorem 4.2 was obtained. The part " (4.4) if and only if $\xi(t)$ and $\eta(t)$ are $M^{+}(2)$-equivalent " of Theorem 4.2 for unit-speed regular curves was obtained in [5, p.136-137, Theorem 5.13]. In these books, the uniqueness of $F$ and the evident form of $F$ are not given.

Definition 4.2. (i) An $I$-path $\xi$ is called a completely degenerate regular path if $g_{\xi}(t)=0$ for all $t \in I$.

(ii) An $I$-path $\xi$ is called a non-degenerate path if $g_{\xi}(t) \neq 0$ for all $t \in I$.

By this definition, it is obvious that every non-degenerate path is regular. If $\xi(t)$ and $\eta(t)$ be $I$-paths in $E_{2}$ such that $\xi(t)$ is completely degenerate and $\xi(t) \stackrel{M(2)}{\sim} \eta(t)$, then $\eta(t)$ is also completely degenerate. Similarly, it is obvious that if $\xi(t)$ is non-degenerate and $\xi(t) \stackrel{M(2)}{\sim} \eta(t)$, then $\eta(t)$ is also non-degenerate.

Theorem 4.3. Let $\xi$ and $\eta$ be two completely degenerate regular I-paths.

$$
\xi \stackrel{M(2)}{\sim} \eta \Leftrightarrow f_{\xi}(t)=f_{\eta}(t)
$$

for all $t \in I$.

Moreover, there exist only $F_{1}, F_{2} \in M^{+}(2)$ such that $\eta(t)=F_{1} \xi(t)=N_{1} \xi(t)+b_{1}$ or $\eta(t)=F_{2} \xi(t)=N_{2} \xi(t)+b_{2}$. Then

(i) in the case $\eta(t)=F_{1} \xi(t)=N_{1} \xi(t)+b_{1}, N_{1} \in O^{+}(2)$ and $b_{1} \in E_{2}$ which are not depend on $t$ in $I$ have the forms (4.2) and

$$
b_{1}=\eta(t)-N_{1} \xi(t)
$$

, resp.

(ii) in the case $\eta(t)=F_{2} \xi(t)=N_{2} \xi(t)+b_{2}, N_{2} \in O^{-}(2)$ and $b_{2} \in E_{2}$ which are not depend on $t$ in $I$ have the forms

$$
N_{2}=\left(\begin{array}{cc}
\frac{\left\langle\bar{\xi}^{\prime}(t), \eta^{\prime}(t)>\right.}{f_{\xi(t)}(t)} & \frac{\left[\bar{\xi}^{\prime}(t) \eta^{\prime}(t)\right]}{f_{\xi}(t)} \\
\frac{\left[\bar{\xi}^{\prime}(t) \eta^{\prime}(t)\right]}{f_{\xi}(t)} & -\frac{<\bar{\xi}^{\prime}(t), \eta^{\prime}(t)>}{f_{\xi}(t)}
\end{array}\right) .
$$

and

$$
b_{2}=\eta(t)-N_{2} \xi(t)
$$

for all $t \in I$, resp.

Proof. A proof of this theorem is obtained from Proposition 4.1 and [15, Theorem 3.6].

Theorem 4.4. Let $\xi(t)$ and $\eta(t)$ be two non-degenerate I-paths in $E_{2}$. Then

$$
\xi \stackrel{M(2)}{\sim} \eta \Leftrightarrow\left\{\begin{array}{l}
f_{\xi}(t)=f_{\eta}(t) \\
h_{\xi}(t)=h_{\eta}(t)
\end{array}\right.
$$

for all $t \in I$.

Moreover, there exists the unique $F \in M(2)$ such that $\eta(t)=F \xi(t)$. In this case there are the following statements:

$\left(i_{1}\right) g_{\xi}(t)=g_{\eta}(t)$ for all $t \in I$.

$\left(i_{2}\right) g_{\xi}(t)=-g_{\eta}(t)$ for all $t \in I$. 
In the case $\left(i_{1}\right), F$ has the form $F \xi(t)=N_{1} \xi(t)+b_{1}$, where $N_{1} \in O^{+}(2)$ and $b_{1} \in E_{2}$ which are not depend on $t$ in $I$ can be written as (4.2) and (4.3), resp.

In the case $\left(i_{2}\right), F$ has the form $F \xi(t)=N_{2} \xi(t)+b_{2}$, where $N_{2} \in O(2)$ and $b_{2} \in E_{2}$ which are not depend on $t$ in $I$ can be written as (4.7) and (4.8),resp.

Proof. A proof of this theorem is obtained from Proposition 4.1 and [15, Theorem 3.7].

Definition 4.3. (see [1, p.31], [20, p.21]) The function $\kappa(\xi)=\frac{\left|g_{\xi}(t)\right|}{f_{\xi}(t)^{\frac{3}{2}}}$ is called the curvature of a regular path $\xi(t)$ in $E_{2}$.

We note that an $I$-path $\xi(t)$ in $E_{2}$ is completely degenerate if and only if $\kappa(\xi)=0$ for all $t \in I$. Similarly, an $I$-path $\xi(t)$ in $E_{2}$ is non-degenerate if and only if $\kappa(\xi) \neq 0$ for all $t \in I$.

Theorem 4.5. Let $\xi(t)$ and $\eta(t)$ be two non-degenerate I-paths in $E_{2}$. Then

$$
\xi \stackrel{M(2)}{\sim} \eta \Leftrightarrow\left\{\begin{array}{c}
f_{\xi}(t)=f_{\eta}(t) \\
\kappa(\xi)=\kappa(\eta)
\end{array}\right.
$$

for all $t \in I$.

Moreover, there exists the unique $F \in M(2)$ such that $\eta(t)=F \xi(t)$. In this case there are the following statements:

$\left(i_{1}\right) g_{\xi}(t)=g_{\eta}(t)$ for all $t \in I$.

$\left(i_{2}\right) g_{\xi}(t)=-g_{\eta}(t)$ for all $t \in I$.

In the case $\left(i_{1}\right), F$ has the form $F \xi(t)=N_{1} \xi(t)+b_{1}$, where $N_{1} \in O^{+(2)}$ and $b_{1} \in E_{2}$ which are not depend on $t$ in $I$ can be written as (4.2) and (4.3), resp.

In the case $\left(i_{2}\right), F$ has the form $F \xi(t)=N_{2} \xi(t)+b_{2}$, where $N_{2} \in O(2)$ and $b_{2} \in E_{2}$ which are not depend on $t$ in $I$ can be written as (4.7) and (4.8),resp.

Proof. Assume that the paths $\xi(t)$ and $\eta(t)$ are $M(2)$-equivalent. Since functions $f_{\xi}(t)=<\xi^{\prime}(t), \xi^{\prime}(t)>$ and $\kappa(\xi)$ are $M(2)$-invariant, equalities (4.10) hold.

Conversely, assume that (4.10) holds. Using equalities implies $g_{\xi}^{2}(t)=\kappa_{ \pm}^{2}(\xi) f_{\xi}(t)^{3}, g_{\eta}^{2}(t)=\kappa_{ \pm}^{2}(\eta) f_{\eta}(t)^{3}$ and (4.10), we obtain equalities (4.9). Then the proof follows the proof of Theorem 4.4.

Theorem 4.6. Let $\xi(t)$ and $\eta(t)$ be two non-degenerate I-paths in $E_{2}$. Then

$$
\xi \stackrel{M(2)}{\sim} \eta \Leftrightarrow\left\{\begin{array}{l}
f_{\xi}(t)=f_{\eta}(t) \\
k_{\xi}(t)=k_{\eta}(t)
\end{array}\right.
$$

for all $t \in I$.

Moreover, there exists the unique $F \in M(2)$ such that $\eta(t)=F \xi(t)$. In this case there are the following statements:

$\left(i_{1}\right) g_{\xi}(t)=g_{\eta}(t)$ for all $t \in I$.

$\left(i_{2}\right) g_{\xi}(t)=-g_{\eta}(t)$ for all $t \in I$.

In the case $\left(i_{1}\right), F$ has the form $F \xi(t)=N_{1} \xi(t)+b_{1}$, where $N_{1} \in O^{+(2)}$ and $b_{1} \in E_{2}$ which are not depend on $t$ in $I$ can be written as (4.2) and (4.3), resp.

In the case $\left(i_{2}\right), F$ has the form $F \xi(t)=N_{2} \xi(t)+b_{2}$, where $N_{2} \in O(2)$ and $b_{2} \in E_{2}$ which are not depend on $t$ in $I$ can be written as (4.7) and (4.8), resp.

Proof. A proof of this theorem is obtained from Proposition 4.1 and [15, Theorem 3.9].

Remark 4.3. The part $(4.11) \Leftrightarrow \xi(t) \stackrel{M(2)}{\sim} \eta(t)$ of Theorem 4.6 was obtained in [2, Theorem 4]. 


\section{Determining a plane path from its global differential invariants}

Theorem 5.1. Let $a_{1}, a_{2}: I \rightarrow \mathbb{R}$. Assume that $\xi: I \rightarrow E_{1}^{2}$ be a regular path satisfying the following equalities

$$
\left\{\begin{array}{l}
f_{\xi}(t)=a_{1}(t) \\
g_{\xi}(t)=a_{2}(t)
\end{array}\right.
$$

for all $t \in I$. Then $\xi(t)$ has the form

$$
\xi(t)=e^{i \theta} \int_{t_{0}}^{t} \sqrt{a_{1}(r)} e^{i \int_{r_{0}}^{r} \frac{a_{2}(u)}{a_{1}(u)} d u} d r+c,
$$

where $c \in \mathbb{C}, 0 \leq \theta<2 \pi$ and $t_{0}, r_{0} \in I$.

Conversely, every I-path in the form (5.2) is a regular I-path and satisfies the equalities (5.1).

Proof. $\Rightarrow$ : Let $\xi$ be a regular $I$-path satisfies the equalities (5.1). From $\frac{v}{u}=\frac{\langle u, v\rangle}{\langle u, u\rangle}+i \frac{[u v]}{\langle u, u\rangle}$ in Proposition 2.1, we have for the path $\xi$ :

$$
\frac{\xi^{\prime \prime}}{\xi^{\prime}}=\frac{f_{\xi}^{\prime}(t)}{2 f_{\xi}(t)}+i \frac{g_{\xi}(t)}{f_{\xi}(t)}=\frac{a_{1}^{\prime}(t)}{2 a_{1}(t)}+i \frac{a_{2}(t)}{a_{1}(t)}
$$

From this equality, we have

$$
\xi^{\prime \prime}=\left(\frac{a_{1}^{\prime}(t)}{2 a_{1}(t)}+i \frac{a_{2}(t)}{a_{1}(t)}\right) \xi^{\prime} .
$$

From the equation (5.4), we obtain the following general solution:

$$
\xi(t)=e^{i \theta} \int_{t_{0}}^{t} \sqrt{a_{1}(r)} e^{i \int_{r_{0}}^{r} \frac{a_{2}(u)}{a_{1}(u)} d u} d r+c,
$$

where $c \in \mathbb{C}$ and $t_{0}, r_{0} \in I$.

$\Leftarrow$ : Assume that an $I$-path $\xi$ has the form (5.2). Then we obtain $f_{\xi}(t)=a_{1}(t)$ and $g_{\xi}(t)=a_{2}(t)$. Clearly, $\xi$ is a regular $I$-path.

Example 5.1. Let $a_{1}(t)=4 t^{2}+1, a_{2}(t)=2$ be two real continuous functions on $\mathbb{R}$ and $\xi(0)=1$. For $0 \leq \theta<2 \pi$ and $t_{0}=0=r_{0} \in \mathbb{R}$, the general solution of the equalities (5.1) has the form:

$$
\begin{array}{r}
\xi(t)=e^{i \theta} \int_{t_{0}}^{t} \sqrt{a_{1}(r)} e^{i \int_{r_{0}}^{r} \frac{a_{2}(u)}{a_{1}(u)} d u} d r+c \\
=e^{i \theta} \int_{0}^{t} \sqrt{4 r^{2}+1} e^{i \int_{0}^{r} \frac{2}{4 u^{2}+1} d u} d r+1 \\
=e^{i \theta} \int_{0}^{t} \sqrt{4 r^{2}+1}[\cos (\arctan 2 r)+i \sin (\arctan 2 r)] d r+1 \\
=e^{i \theta} \int_{0}^{t} \sqrt{4 r^{2}+1}\left(\frac{1}{\sqrt{4 r^{2}+1}}+i \frac{2 r}{\sqrt{4 r^{2}+1}}\right) d r+1 \\
=e^{i \theta}\left(t+i t^{2}\right)+1
\end{array}
$$

Since $\xi^{\prime}(t)=e^{i \theta}(1+2 i t) \neq 0, \forall t \in \mathbb{R}, \xi(t)$ is a regular $\mathbb{R}$-path.

Theorem 5.2. Let $a_{1}, a_{3}: I \rightarrow \mathbb{R}$. Assume that $\xi: I \rightarrow E_{1}^{2}$ be a regular path satisfying the following equalities

$$
\left\{\begin{aligned}
f_{\xi}(t) & =a_{1}(t) \\
\kappa_{ \pm}(\xi) & =a_{3}(t)
\end{aligned}\right.
$$

for all $t \in I$. Then $\xi(t)$ has the form

$$
\xi(t)=e^{i \theta} \int_{t_{0}}^{t} \sqrt{a_{1}(r)} e^{i \int_{r_{0}}^{r} a_{3}(u) \sqrt{a_{1}(u)} d u} d r+c,
$$

where $c \in \mathbb{C}, 0 \leq \theta<2 \pi$ and $t_{0}, r_{0} \in I$.

Conversely, every I-path in the form (5.5) is a regular I-path and satisfies the equalities (5.5) for all $t \in I$. 
Proof. The proof is follows from Theorem 4.5 and Theorem 5.1.

Remark 5.1. In [4, Theorem 6.1], the existence theorem was given in the following form: Let $c: I \rightarrow R$ be a smooth function. There exists a regular parametrized curve $z: I \rightarrow E_{2}$ whose associated signed curvature function is $c(t)$. Thus in this book, the existence theorem is given only for the one equation $\kappa_{ \pm}(\xi(t))=c(t)$.

In [5, Theorem 5.14], the existence theorem was given for unit-speed curves (that is $a_{1}(t)=1, \forall t \in I$ in (5.2)) and it is given in the following form: Let $k(s)$ be a real continuous function on $I$. Then there exists a regular parametrized curve $\beta: I \rightarrow E_{2}$ whose associated signed curvature function is $k(s)$ and it has the form

$$
\beta(s)=\left(\int \cos \theta(s) d s+c, \int \sin \theta(s) d s+d\right),
$$

where $\theta(s)=\int k(s) d s+\theta_{0}$ and $c, d, \theta_{0}$ are constants of integration. This path coincides with (5.2) in the case $a(t)=1, \forall t \in I$.

Corollary 5.1. Let $a_{1}: I \rightarrow \mathbb{R}$. Assume that $\xi: I \rightarrow E_{1}^{2}$ be a completely degenerate regular path satisfying the following equalities

$$
f_{\xi}(t)=a(t)
$$

for all $t \in I$. Then $\xi(t)$ has the form

$$
\xi(t)=e^{i \theta} \int_{t_{0}}^{t} \sqrt{a_{1}(r)} d r+c,
$$

where $c \in \mathbb{C}, 0 \leq \theta<2 \pi$ and $t_{0}, r_{0} \in I$.

Conversely, every I-path in the form (5.8) is a completely degenerate regular I-path and satisfies the equality (5.7) for all $t \in I$.

Proof. The proof is obtained from the proof of Theorem 5.1.

Theorem 5.3. Let $a_{1}, a_{4}: I \rightarrow \mathbb{R}$. Assume that $\xi: I \rightarrow E_{1}^{2}$ be a non-degenerate path satisfying the following equalities

$$
\left\{\begin{array}{l}
f_{\xi}(t)=a_{1}(t), \\
h_{\xi}^{2}(t)=a_{4}(t)
\end{array}\right.
$$

for all $t \in I$. Then

(i) For every non-degenerate I-path $\xi$ in $E_{2}, a_{1}(t)>0$ and $a_{4}(t)>0, \forall t \in I$.

(ii) In the case $g_{\xi}(t)>0, \forall t \in I$, $\xi$ has the form

$$
\xi(t)=e^{i \theta} \int_{t_{0}}^{t} \sqrt{a_{1}(r)} e^{i \int_{r_{0}}^{r} \frac{\sqrt{a_{1}(u)}}{a_{1}(u)} d u} d r+c,
$$

where $\forall c \in \mathbb{C}, 0 \leq \theta<2 \pi$ and $t_{0}, r_{0} \in I$.

(iii) In the case $g_{\xi}(t)<0, \forall t \in I$, $\xi$ has the form

$$
\xi(t)=e^{i \theta} \int_{t_{0}}^{t} \sqrt{a_{1}(r)} e^{-i \int_{r_{0}}^{r} \frac{\sqrt{a_{4}(u)}}{a_{1}(u)} d u} d r+c,
$$

where $\forall c \in \mathbb{C}, 0 \leq \theta<2 \pi$ and $t_{0}, r_{0} \in I$.

Conversely, for $a_{4}(t)>0$ for all $t \in I$, every path $\xi$ of the forms (5.10) and (5.11) is a non-degenerate I-path satisfying equalities (5.9).

Proof. Let $\xi: I \rightarrow E_{1}^{2}$ be a non-degenerate $I$-path. Then we have $g_{\xi}(t) \neq 0$ for all $t \in I$. By this inequality and equalities (5.9), we have $a_{4}(t)>0$ for all $t \in I$. Furthermore, since $g_{\xi}(t) \neq 0$ for all $t \in I$, the statements $g_{\xi}(t)>0$ or $g_{\xi}(t)<0$ for all $t \in I$ exist. 
In the case $g_{\xi}(t)>0$,

$$
h_{\xi}^{2}(t)=a_{4}(t)
$$

implies

$$
h_{\xi}(t)=\sqrt{a_{4}(t)} .
$$

Then, by the equality (5.13), we have the system

$$
\left\{\begin{array}{l}
f_{\xi}(t)=a_{1}(t), \\
h_{\xi}(t)=\sqrt{a_{4}(t)}
\end{array}\right.
$$

By Theorem 5.1, a general solution of (5.14) obtain in the form (5.10).

Similarly, in the case $g_{\xi}(t)<0$, the equality (5.12) imply

$$
h_{\xi}(t)=-\sqrt{a_{4}(t)}
$$

Then, we have the system

$$
\left\{\begin{array}{l}
f_{\xi}(t)=a_{1}(t), \\
h_{\xi}(t)=-\sqrt{a_{4}(t)}
\end{array}\right.
$$

By Theorem 5.1, a general solution of (5.15) obtain in the form (5.11).

Conversely, assume that an $I$-path $\xi$ have the forms (5.10) or (5.11). Then, we obtain equalities (5.9). However since $a_{4}(t) \neq 0$ for all $t \in I$, clearly $\xi$ is a non-degenerate $I$-path.

Theorem 5.4. Let $a_{1}, a_{3}: I \rightarrow \mathbb{R}$. Assume that $\xi: I \rightarrow E_{1}^{2}$ be a regular path satisfying the following equalities

$$
\left\{\begin{aligned}
f_{\xi}(t) & =a_{1}(t) \\
\kappa_{ \pm}(\xi) & =a_{5}(t)
\end{aligned}\right.
$$

for all $t \in I$. Then

(i) For every non-degenerate I-path $\xi$ in $E_{2}, a_{1}(t)>0$ and $a_{5}(t)>0, \forall t \in I$.

(ii) For $g_{\xi}(t)>0, \forall t \in I$, $\xi$ has the form

$$
\xi(t)=e^{i \theta} \int_{t_{0}}^{t} \sqrt{a_{1}(r)} e^{i \int_{r_{0}}^{r} a_{5}(u) \sqrt{a_{1}(u)} d u} d r+c,
$$

where $\forall c \in \mathbb{C}, 0 \leq \theta<2 \pi$ and $t_{0}, r_{0} \in I$.

(iii) For $g_{\xi}(t)<0, \forall t \in I$, $\xi$ has the form

$$
\xi(t)=e^{i \theta} \int_{t_{0}}^{t} \sqrt{a_{1}(r)} e^{-i \int_{r_{0}}^{r} a_{5}(u) \sqrt{a_{1}(u)} d u} d r+c,
$$

where $\forall c \in \mathbb{C}, 0 \leq \theta<2 \pi$ and $t_{0}, r_{0} \in I$.

Conversely, every path $\xi$ of the forms (5.17) and (5.18) is a non-degenerate I-path satisfying equalities (5.16).

Proof. The proof is similar to the proof of Theorem 5.3.

Theorem 5.5. Let $a_{1}, a_{3}: I \rightarrow \mathbb{R}$. Assume that $\xi: I \rightarrow E_{1}^{2}$ be a non-degenerate path satisfying the following equalities

$$
\left\{\begin{array}{l}
f_{\xi}(t)=a_{1}(t), \\
k_{\xi}(t)=a_{6}(t)
\end{array}\right.
$$

for all $t \in I$. Then 
(i) $4 a_{1}(t) a_{6}(t)-\left(a_{1}^{\prime}(t)\right)^{2}>0$ for all $t \in I$,

(ii) For $g_{\xi}(t)>0, \forall t \in I$, $\xi$ has the form

$$
\xi(t)=e^{i \theta} \int_{t_{0}}^{t} \sqrt{a_{1}(r)} e^{i \int_{r_{0}}^{r} \frac{\sqrt{a_{1}(u) a_{6}(u)-\left(a_{1}^{\prime}(u)\right)^{2}}}{a_{1}(u)}} d u d r+c,
$$

where $\forall c \in \mathbb{C}, 0 \leq \theta<2 \pi$ and $t_{0}, r_{0} \in I$.

(iii) For $g_{\xi}(t)<0, \forall t \in I$, $\xi$ has the form

$$
\xi(t)=e^{i \theta} \int_{t_{0}}^{t} \sqrt{a_{1}(r)} e^{-i \int_{r_{0}}^{r} \frac{\sqrt{a_{1}(u) a_{6}(u)-\left(a_{1}^{\prime}(u)\right)^{2}}}{a_{1}(u)} d u} d r+c,
$$

where $\forall c \in \mathbb{C}, 0 \leq \theta<2 \pi$ and $t_{0}, r_{0} \in I$.

Conversely, in the case $4 a_{1}(t) a_{6}(t)-\left(a_{1}^{\prime}(t)\right)^{2}>0$, every path $\xi$ of the forms (5.20) and (5.21) is a non-degenerate I-path satisfying equalities (5.19).

Proof. The proof is obtained from Theorem 5.4.

\section{Equivalence conditions of regular curves}

Now, we will give the conditions of the global $G$-equivalence of regular curves in terms of the type and global differential $G$-invariants of a regular curve for the groups $G=M(2), M^{+}(2)$.

By Theorem 3.1, G-equivalence and uniqueness problems for curves are reduced to the same problems for invariant parametrizations of curves only for the case $L_{\Phi}=L_{\Psi} \neq(-\infty,+\infty)$.

Let $\Phi$ be regular curves and $\xi \in P_{\Phi}$ be an invariant parametrization.

We consider, for all $s \in L_{\Phi}$, the functions $f_{\xi}(s), g_{\xi}(s), h_{\xi}(s)$ and $k_{\xi}(s)$ in Proposition 4.2.

Theorem 6.1. Let $\Phi$ and $\Psi$ are regular curves such that $L_{\Phi} \neq(-\infty,+\infty), L_{\Psi} \neq(-\infty,+\infty)$ and $\xi \in P_{\Phi}, \eta \in P_{\Psi}$ are invariant parametrizations. Then $\Phi$ and $\Psi$ are $M^{+}(2)$-equivalent if and only if

$$
\left\{\begin{array}{c}
L_{\Phi}=L_{\Psi} \\
g_{\xi}(s)=g_{\eta}(s)
\end{array}\right.
$$

for all $s \in L_{\Phi}$.

Moreover, there is the unique $F \in M^{+}(2)$ such that $\Psi=F \Phi=R_{1} \Phi+c_{1}$, where $R_{1} \in O^{+}(2)$ and $c_{1} \in E_{2}$ can be written as

$$
R_{1}=\left(\begin{array}{cc}
<\xi^{\prime}(s), \eta^{\prime}(s)> & -\left[\xi^{\prime}(s) \eta^{\prime}(s)\right] \\
{\left[\xi^{\prime}(s) \eta^{\prime}(s)\right]} & <\xi^{\prime}(s), \eta^{\prime}(s)>
\end{array}\right)
$$

and

$$
c_{1}=\eta(s)-R_{1} \xi(s)
$$

for all $s \in L_{\Phi}$, resp. However, $R_{1}$ and $c_{1}$ are not depend on $s \in L_{\Phi}$.

Proof. $\Rightarrow$ : Let $\Phi$ and $\Psi$ be $M^{+}(2)$-equivalent. Using Proposition 3.3, we have $L_{\Phi}=L_{\Psi}$. Hence, by $L_{\Phi}=L_{\Psi}$ and Theorem 3.1, we have $\xi$ and $\eta$ are $M^{+}(2)$-equivalent. By Theorem 4.1 , for all $s \in L_{\Phi}$, we obtain $f_{\xi}(s)=f_{\eta}(s)$ and $g_{\xi}(s)=g_{\eta}(s)$.

Since $\xi$ and $\eta$ are regular curves, with using $g_{\xi}(s)=g_{\eta}(s)$, the equalities (6.1) hold.

$\Leftarrow$ : Let $L_{\Phi}=L_{\Psi}, g_{\xi}(s)=g_{\eta}(s)$ for all $s \in L_{\Phi}$. Since $\xi \in P_{\Phi}, \eta \in P_{\Psi}$, by Proposition 3.5 , we have $f_{\xi}(s)=f_{\eta}(s)=$ 1 for all $s \in L_{\Phi}$. Using this equality and $g_{\xi}(s)=g_{\eta}(s)$, we obtain the equalities (4.1).

By Theorem 4.1, we obtain that $\xi$ and $\eta$ are $M^{+}(2)$-equivalent. Then, there is the unique $F \in M^{+}(2)$ such that $\eta(s)=F \xi(s)=R_{1} \xi(s)+c_{1}$, where $R_{1} \in O^{+}(2)$ and $c_{1} \in E_{2}$. Then $R_{1}$ and $c_{1}$ have the forms (6.2) and (6.4), respectively. Here $R_{1}$ and $c_{1}$ are not depend on $s$ in $L_{\Phi}$. From $\xi \in P_{\Phi}, \eta \in P_{\Psi}$, Theorem 3.1 and $\eta=F \xi$, we have $\Psi=F \Phi$. 
Remark 6.1. Theorem 6.1 shows that the system $\left\{L_{\Phi}, g_{\xi}(s)\right\}$ is a complete system of invariants of a curve $\Phi$ for the case $L_{\Phi} \neq(-\infty,+\infty)$ and $\xi(s) \in \Phi$. In the case $L_{\Phi}=(-\infty,+\infty)$, they are not invariants of a curve $\Phi$.

Theorem 6.2. Let $\Phi$ and $\Psi$ are regular curves such that $L_{\Phi}=L_{\Psi}=(-\infty,+\infty)$ and $\xi \in P_{\Phi}, \eta \in P_{\Psi}$ are invariant parametrizations. Then $\Phi$ and $\Psi$ are $M^{+}(2)$-equivalent if and only if there exists $c \in L_{\Phi}$ such that the following equalities

$$
\left\{g_{\xi}(s+c)=g_{\eta}(s)\right.
$$

hold for all $s \in L_{\Phi}$.

Moreover, there is the unique $F \in M^{+}(2)$ such that $\Psi=F \Phi=R_{2} \Phi+c_{2}$, where $R_{2} \in O^{+}(2)$ and $c_{2} \in E_{2}$ can be written as

$$
R_{2}=\left(\begin{array}{cc}
<\xi^{\prime}(s+c), \eta^{\prime}(s)> & -\left[\xi^{\prime}(s+c) \eta^{\prime}(s)\right] \\
{\left[\xi^{\prime}(s+c) \eta^{\prime}(s)\right]} & <\xi^{\prime}(s+c), \eta^{\prime}(s)>
\end{array}\right)
$$

and

$$
c_{2}=\eta(s)-N_{2} \xi(s),
$$

respectively. Here $R_{2}$ and $c_{2}$ are not depend on $s$ in $L_{\Phi}$.

Proof. $\Rightarrow$ : Let $\Phi$ and $\Psi$ be $M^{+}(2)$-equivalent. Using Proposition 3.3, we have $L_{\Phi}=L_{\Psi}$. Hence, by $L_{\Phi}=L_{\Psi}$ and Theorem 3.1, there exits $c \in(-\infty,+\infty)$ such that $\xi(s+c)$ and $\eta(s)$ are $M^{+}(2)$-equivalent. By Theorem 4.2, for all $s \in L_{\Phi}$, we obtain $f_{\xi}(s+c)=f_{\eta}(s)$ and $\kappa_{ \pm}(\xi)(s+c)=\kappa_{ \pm}(\eta)(s)$.

Since $\xi$ and $\eta$ are regular, with using $\kappa_{ \pm}(\xi)(s+c)=\kappa_{ \pm}(\eta)(s)$, we obtain the equalities (6.4).

$\Leftarrow$ : Let $L_{\Phi}=L_{\Psi}, f_{\xi}(s+c)=f_{\eta}(s)$ for all $s \in L_{\Phi}$ and for some $c \in L_{\Phi}$. Since $\xi \in P_{\Phi}, \eta \in P_{\Psi}$, by Proposition 3.5 , we have $f_{\xi}(s)=f_{\eta}(s)=1$ for all $s \in L_{\Phi}$. Using this equality, we have $f_{\xi}(s+c)=f_{\eta}(s)$ for all $s \in L_{\Phi}$. From $g_{\xi}(s+c)=g_{\eta}(s)$ and (6.4), we have (4.1). By Theorem 4.2, we obtain that $\xi$ and $\eta$ are $M^{+}(2)$-equivalent.

Then, there exists the unique $F \in M^{+}(2)$ such that $\eta(s)=F \xi(s+c)=R_{2} \xi(s+c)+c_{2}$, where $R_{2} \in O^{+}(2)$ and $c_{2} \in E_{2}$. Then $R_{2}$ and $c_{2}$ have the forms (6.5) and (6.6), respectively. Here $R_{2}$ and $c_{2}$ do independent of the choice of $s \in L_{\Phi}$. From $\xi \in P_{\Phi}, \eta \in P_{\Psi}$, Theorem 3.1 and $\eta(s)=F \xi(s+c)$, we have $\Psi=F \Phi$.

Proposition 6.1. Let $\Phi$ be a regular curve in $E_{2}$ and $\xi \in P_{\Phi}$. Then $\kappa_{ \pm}(\xi(s))=g_{\xi}(s)$ for all $s \in L_{\Phi}$.

Proof. Since $\xi \in P_{\Phi}$, we have $f_{\xi}(s)=1$ for all $s \in L_{\Phi}$. This equality and the equality $\kappa_{ \pm}(\xi(s))=\frac{g_{\xi}(s)}{f_{\xi}(s)^{\frac{3}{2}}}$ for all $s \in L_{\Phi}$ implies the equality $\kappa_{ \pm}(\xi(s))=g_{\xi}(s)$ for all $s \in L_{\Phi}$.

Theorem 6.3. Let $\Phi$ and $\Psi$ are regular curves such that $L_{\Phi} \neq(-\infty,+\infty), L_{\Psi} \neq(-\infty,+\infty)$ and $\xi \in P_{\Phi}, \eta \in P_{\Psi}$ are invariant parametrizations. Then $\Phi$ and $\Psi$ are $M^{+}(2)$-equivalent if and only if

$$
\left\{\begin{aligned}
L_{\Phi} & =L_{\Psi} \\
\kappa_{ \pm}(\xi(s)) & =\kappa_{ \pm}(\eta(s))
\end{aligned}\right.
$$

for all $s \in L_{\Phi}$.

Furthermore, if $\Phi$ and $\Psi$ are $M^{+}(2)$-equivalent, there is the unique $F \in M^{+}(2)$ such that $\Psi=F \Phi=R_{3} \Phi+c_{3}$, where $R_{3} \in O^{+}(2)$ and $c_{3} \in E_{2}$ have the forms (6.2) and (6.4),resp.

Proof. It follows from Proposition3.3 Theorems 3.1, 4.2 and 6.1.

Theorem 6.4. Let $\Phi$ and $\Psi$ are regular curves such that $L_{\Phi}=L_{\Psi}=(-\infty,+\infty)$ and $\xi \in P_{\Phi}, \eta \in P_{\Psi}$ are invariant parametrizations. Then $\Phi$ and $\Psi$ are $M^{+}(2)$-equivalent if and only if there exists $c \in L_{\Phi}$ such that the equality

$$
\kappa_{ \pm}(\xi(s+c))=\kappa_{ \pm}(\eta(s))
$$

holds for all $s \in L_{\Phi}$.

Furthermore, if $\Phi$ and $\Psi$ are $M^{+}(2)$-equivalent, there is the unique $F \in M^{+}(2)$ such that $\Psi=F \Phi=R_{4} \Phi+c_{4}$, where $R_{4} \in O^{+}(2)$ and $c_{4} \in E_{2}$ have the forms (6.5) and (6.6),resp.

Proof. It follows from Proposition3.3 Theorems 3.1, 4.2 and 6.2. 
Definition 6.1. A curve $\Phi$ is called completely degenerate if it contains a completely degenerate path. A curve $\Phi$ is called non-degenerate if it contains a non-degenerate path.

Proposition 6.2. Let $\Phi$ be a completely degenerate curve or a non-degenerate curve. Then every parametrization $\xi \in \Phi$ is a completely degenerate regular path or a non-degenerate path.

Proof. It is similar to the proof of [15, Proposition 5.3].

Theorem 6.5. Let $\Phi$ and $\Psi$ are completely degenerate regular curves such that $L_{\Phi} \neq(-\infty,+\infty), L_{\Psi} \neq(-\infty,+\infty)$ and $\xi \in P_{\Phi}, \eta \in P_{\Psi}$ are invariant parametrizations. Then $\Phi$ and $\Psi$ are $M(2)$-equivalent if and only if

$$
L_{\Phi}=L_{\Psi}
$$

for all $s \in L_{\Phi}$.

Furthermore, there are only two $F_{1}, F_{2} \in M(2)$ such that $\Psi=F_{1} \Phi=T_{1} \Phi+d_{1}$ or $\Psi=F_{2} \Phi=T_{2} \Phi+d_{2}$. Then

(i) in the case $\Psi=F_{1} \Phi=T_{1} \Phi+d_{1}$, the element $T_{1} \in O^{+}(2)$ and $d_{1} \in E_{2}$ can be written as (6.2) and (6.4), resp.

(ii) in the case $\Psi=F_{2} \Phi=T_{2} \Phi+d_{2}$, the element $T_{2} \in O^{-}(2)$ and $d_{2} \in E_{2}$ can be written as

$$
T_{2}=\left(\begin{array}{cc}
<\bar{\xi}^{\prime}(s), \eta^{\prime}(s)> & {\left[\bar{\xi}^{\prime}(s) \eta^{\prime}(s)\right]} \\
{\left[\bar{\xi}^{\prime}(s) \eta^{\prime}(s)\right]} & -<\bar{\xi}^{\prime}(s), \eta^{\prime}(s)>
\end{array}\right) .
$$

and

$$
d_{2}=\eta(s)-T_{2} \xi(s)
$$

for all $s \in L_{\Phi}$. Here $T_{1}, T_{2}, d_{1}$ and $d_{2}$ are not depend on $s \in L_{\Phi}$.

Proof. It follows from Theorems 4.3 and 6.1.

Theorem 6.6. Let $\Phi$ and $\Psi$ are completely degenerate regular curves such that $L_{\Phi}=L_{\Psi}=(-\infty,+\infty)$. Then $\Phi$ and $\Psi$ are $M(2)$-equivalent.

Proof. A proof follows easy from Theorems 3.1, 4.3 and 6.2.

Theorem 6.7. Let $\Phi$ and $\Psi$ are non-degenerate curves such that $L_{\Phi} \neq(-\infty,+\infty), L_{\Psi} \neq(-\infty,+\infty)$ and $\xi \in P_{\Phi}, \eta \in$ $P_{\Psi}$ are invariant parametrizations. Then $\Phi$ and $\Psi$ are $M(2)$-equivalent if and only if

$$
\begin{array}{cc}
L_{\Phi} & =L_{\Psi} \\
h_{\xi}(s) & =h_{\eta}(s)
\end{array}
$$

for all $s \in L_{\Phi}$.

Furthermore, if $\Phi$ and $\Psi$ are $M(2)$-equivalent, there is the unique $F \in M(2)$ such that $\Psi=F \Phi$ for all $s \in L_{\Phi}$. Then

(i) in the case $g_{\xi}(s)=g_{\eta}(s), F$ has the form $\Psi=F \Phi=U_{1} \Phi+k_{1}$, where the $U_{1} \in O^{+}(2)$ and $k_{1} \in E_{2}$ have the forms (6.2) and (6.4),resp.

(ii) in the case $g_{\xi}(s)=-g_{\eta}(s), F$ has the form $\Psi=F \Phi=U_{2} \Phi+k_{2}$, where $U_{2} \in O(2)$ and $k_{2} \in E_{2}$ have the forms (6.10) and (6.11), resp.

Here $U_{1}, U_{2}, k_{1}$ and $k_{2}$ are not depend on $s \in L_{\Phi}$.

Proof. It follows from Theorems 3.1,4.4 and 6.1.

Theorem 6.8. Let $\Phi$ and $\Psi$ are non-degenerate curves such that $L_{\Phi}=L_{\Psi}=(-\infty,+\infty)$ and $\xi \in P_{\Phi}, \eta \in P_{\Psi}$ are invariant parametrizations. Then $\Phi$ and $\Psi$ are $M(2)$-equivalent if and only if there exists $c \in L_{\Phi}$ such that the equality

$$
g_{\xi}(s+c)=g_{\eta}(s)
$$

holds for all $s \in L_{\Phi}$.

Furthermore, if $\Phi$ and $\Psi$ are $M(2)$-equivalent, there is the unique $F \in M(2)$ such that $\Psi=F \Phi$, then 
(i) in the case $g_{\xi}(s+c)=g_{\eta}(s), F$ has the form $\Psi=F \Phi=U_{1} \Phi+k_{1}$, where $U_{1} \in O^{+}(2)$ and $k_{1} \in E_{2}$ have the forms (6.5) and (6.6), resp.

(ii) in the case $g_{\xi}(s)=-g_{\eta}(s), F$ has the form $\Psi=F \Phi=U_{2} \Phi+k_{2}$, where $U_{2} \in O(2)$ and $k_{2} \in E_{2}$ have the forms

$$
U_{2}=\left(\begin{array}{cc}
<\bar{\xi}^{\prime}(s+c), \eta^{\prime}(s)> & {\left[\bar{\xi}^{\prime}(s+c) \eta^{\prime}(s)\right]} \\
{\left[\bar{\xi}^{\prime}(s+c) \eta^{\prime}(s)\right]} & -<\bar{\xi}^{\prime}(s+c), \eta^{\prime}(s)>
\end{array}\right)
$$

and

$$
k_{2}=\eta(s)-T_{2} \xi(s+c)
$$

for all $s \in L_{\Phi}$, resp.

Proof. It follows from Theorems 3.1,4.4 and 6.2.

Proposition 6.3. Let $\Phi$ be a non-degenerate curve in $E_{2}$ and $\xi \in P_{\Phi}$. Then $\kappa(\xi)=\left|g_{\xi}(s)\right|$ for all $s \in L_{\Phi}$.

Proof. Since $\xi \in P_{\Phi}$, we have $f_{\xi}(s)=1$ for all $s \in L_{\Phi}$. This equality and the equality $\kappa(\xi)=\frac{\left|g_{\xi}(s)\right|}{f_{\xi}(s)^{\frac{3}{2}}}$ for all $s \in L_{\Phi}$ implies the equality $\kappa(\xi)=\left|g_{\xi}(s)\right|$ for all $s \in L_{\Phi}$.

Theorem 6.9. Let $\Phi$ and $\Psi$ are non-degenerate curves such that $L_{\Phi} \neq(-\infty,+\infty), L_{\Psi} \neq(-\infty,+\infty)$ and $\xi \in P_{\Phi}, \eta \in$ $P_{\Psi}$ are invariant parametrizations. Then $\Phi$ and $\Psi$ are $M(2)$-equivalent if and only if

$$
\begin{array}{rc}
L_{\Phi} & =L_{\Psi} \\
\kappa(\xi(s)) & =\kappa(\eta(s))
\end{array}
$$

for all $s \in L_{\Phi}$.

Furthermore, if $\Phi$ and $\Psi$ are $M(2)$-equivalent, there is the unique $F \in M(2)$ such that $\Psi=F \Phi$ for all $s \in L_{\Phi}$. Then

(i) in the case $g_{\xi}(s)=g_{\eta}(s), F$ has the form $\Psi=F \Phi=U_{1} \Phi+k_{1}$, where $U_{1} \in O^{+}(2)$ and $k_{1} \in E_{2}$ have the forms (6.2) and (6.4), resp.

(ii) in the case $g_{\xi}(s)=-g_{\eta}(s), F$ has the form $\Psi=F \Phi=U_{2} \Phi+k_{2}$, where $U_{2} \in O(2)$ and $k_{2} \in E_{2}$ have the forms (6.10) and (6.11), resp.

Here $U_{1}, U_{2}, k_{1}$ and $k_{2}$ are not depend on $s \in L_{\Phi}$.

Proof. It follows from Theorems 3.1,4.5 and 6.7.

Theorem 6.10. Let $\Phi$ and $\Psi$ are non-degenerate curves such that $L_{\Phi}=L_{\Psi}=(-\infty,+\infty)$ and $\xi \in P_{\Phi}, \eta \in P_{\Psi}$ are invariant parametrizations. Then $\Phi$ and $\Psi$ are $M(2)$-equivalent if and only if there exists $c \in L_{\Phi}$ such that the equality

$$
\kappa(\xi(s+c))=\kappa(\eta(s)))
$$

holds for all $s \in L_{\Phi}$.

Furthermore, if $\Phi$ and $\Psi$ are $M(2)$-equivalent, there is the unique $F \in M(2)$ such that $\Psi=F \Phi$. Then

(i) in the case $g_{\xi}(s+c)=g_{\eta}(s)$, $F$ has the form $\Psi=F \Phi=U_{1} \Phi+k_{1}$, where $U_{1} \in O^{+}(2)$ and $k_{1} \in E_{2}$ have the forms (6.5) and (6.6),resp.

(ii) in the case $g_{\xi}(s)=-g_{\eta}(s), F$ has the form $\Psi=F \Phi=U_{2} \Phi+k_{2}$, where $U_{2} \in O(2)$ and $k_{2} \in E_{2}$ have the forms (6.15) and (6.16), resp.

Proof. It follows from Proposition 6.3, Theorems 3.1,4.5 and 6.8.

Theorem 6.11. Let $\Phi$ and $\Psi$ are non-degenerate curves such that $L_{\Phi} \neq(-\infty,+\infty), L_{\Psi} \neq(-\infty,+\infty)$ and $\xi \in P_{\Phi}, \eta \in$ $P_{\Psi}$ are invariant parametrizations. Then $\Phi$ and $\Psi$ are $M(2)$-equivalent if and only if

$$
\begin{gathered}
L_{\Phi}=L_{\Psi} \\
k_{\xi}(s)=k_{\eta}(s)
\end{gathered}
$$

for all $s \in L_{\Phi}$.

Furthermore, if $\Phi$ and $\Psi$ are $M(2)$-equivalent, there is the unique $F \in M(2)$ such that $\Psi=F \Phi$ for all $s \in L_{\Phi}$. Then 
(i) in the case $g_{\xi}(s)=g_{\eta}(s), F$ has the form $\Psi=F \Phi=U_{1} \Phi+k_{1}$, where $U_{1} \in O^{+}(2)$ and $k_{1} \in E_{2}$ have the forms (6.2) and (6.4), resp.

(ii) in the case $g_{\xi}(s)=-g_{\eta}(s), F$ has the form $\Psi=F \Phi=U_{2} \Phi+k_{2}$, where $U_{2} \in O(2)$ and $k_{2} \in E_{2}$ have the forms (6.10) and (6.11), resp.

Here $U_{1}, U_{2}, k_{1}$ and $k_{2}$ are not depend on $s \in L_{\Phi}$.

Proof. It follows from Theorems 3.1, 4.6 and 6.1.

Theorem 6.12. Let $\Phi$ and $\Psi$ are non-degenerate curves such that $L_{\Phi}=L_{\Psi}=(-\infty,+\infty)$ and $\xi \in P_{\Phi}, \eta \in P_{\Psi}$ are invariant parametrizations. Then $\Phi$ and $\Psi$ are $M(2)$-equivalent if and only if there exists $c \in L_{\Phi}$ such that the equality

$$
k_{\xi}(s+c)=k_{\eta}(s)
$$

holds for all $s \in L_{\Phi}$.

Furthermore, if $\Phi$ and $\Psi$ are $M(2)$-equivalent, there is the unique $F \in M(2)$ such that $\Psi=F \Phi$. Then

(i) in the case $g_{\xi}(s+c)=g_{\eta}(s), F$ has the form $\Psi=F \Phi=U_{1} \Phi+k_{1}$, where $U_{1} \in O^{+}(2)$ and $k_{1} \in E_{2}$ have the forms (6.5) and (6.6), resp.

(ii) in the case $g_{\xi}(s)=-g_{\eta}(s), F$ has the form $\Psi=F \Phi=U_{2} \Phi+k_{2}$, where $U_{2} \in O(2)$ and $k_{2} \in E_{2}$ have the forms (6.15) and (6.16), resp.

Proof. It follows from Theorems 3.1, 4.6 and 6.2.

\section{Existence and rigidity theorems for regular curves}

By Theorem 3.1, the existence problem for curves is reduced to the same problem for invariant parametrizations of curves. Below we use this reduction.

Theorem 7.1. Let $\Phi$ be a regular curve in $E_{2}, \xi(s) \in P_{\Phi}$ and $b(s)$ be a real continuous function on $L_{\Phi}$. Let $c \in L_{\Phi}$, $h \in E_{2}$ be given. Then every $\xi(s) \in P_{\Phi}$ such that $\xi(c)=h$ and $\left[\xi^{\prime}(s) \xi^{\prime \prime}(s)\right]=b(s)$ has the following form

$$
\xi(s)=h+e^{i \varphi} \int_{c}^{s} e^{i \int_{v_{0}}^{v} b(u) d u} d v,
$$

where $\varphi \in \mathbb{R}, v_{0} \in L_{\Phi}$. Conversely, every path $\xi(s)$ of the form (7.1) is an invariant parametrization of a regular curve in $E_{2}$.

Proof. By Proposition 3.5, an invariant parametrization $\xi(s)$ of a regular curve $\Phi$ satisfies the condition $<\xi^{\prime}(s), \xi^{\prime}(s)>=a(s)=1$ for all $s \in L_{\Phi}$. Using this equality and (5.1), we obtain (7.1). Conversely, (7.1) implies $\xi^{\prime}(s)=e^{i \varphi} e^{i \int_{s_{0}}^{s} b(u) d u}$. It is easy to see $\left\langle\xi^{\prime}(s), \xi^{\prime}(s)>=1\right.$. Hence (7.1) is an invariant parametrization of the curve $\Phi$.

Theorem 7.2. Let $\Phi$ be a regular curve in $E_{2}, \xi(s) \in P_{\Phi}$ and $b(s)$ be a real continuous function on $L_{\Phi}$. Let $s_{0} \in L_{\Phi}$, $h \in E_{2}$ be given. Then every $\xi(s) \in P_{\Phi}$ such that $x\left(s_{0}\right)=h$ and $\kappa_{ \pm}(\xi(s))=b(s)$ has the form (7.1). Conversely, every path $\xi(s)$ of the form (7.1) is an invariant parametrization of a regular curve in $E_{2}$.

Proof. It follows from Theorem 5.1 by using the equality $\left\langle\xi^{\prime}(s), \xi^{\prime}(s)>=1\right.$ for all $s \in L_{\Phi}$.

Theorem 7.3. Let $\Phi$ be a completely degenerate regular curve in $E_{2}$. Let $c \in L_{\Phi}, h \in E_{2}$ be given. Then every $\xi(s) \in P_{\Phi}$ such that $\xi(c)=h$ has the following form

$$
\xi(s)=h+e^{i \varphi}(s-c),
$$

where $\varphi \in \mathbb{R}$. Conversely, every path $\xi(s)$ of the form (7.2) is an invariant parametrization of a completely degenerate regular curve in $E_{2}$.

Proof. Since $<\xi^{\prime}(s), \xi^{\prime}(s)>=1$ and $\left[\xi^{\prime}(s) \xi^{\prime \prime}(s)\right]=b(s)=0$ for the invariant parametrization $\xi(s)$ of a completely degenerate regular curve, a proof follows from Theorem 7.1. 
Theorem 7.4. Let $\Phi$ be a non-degenerate curve in $E_{2}, \xi(s) \in P_{\Phi}$ and $c(s)$ be a real continuous function on $L_{\Phi}$ such that $c(s)>0$ for all $s \in L_{\Phi}$. Let $c \in L_{\Phi}, h \in E_{2}$ be given. Assume that $\xi(s) \in P_{\Phi}$ such that $\xi(c)=h$ and $\left[\xi^{\prime}(s) \xi^{\prime \prime}(s)\right]^{2}=c(s)$. Then the following cases exist:

(i) $\left[\xi^{\prime}(s) \xi^{\prime \prime}(s)\right]=\sqrt{c(s)}$

(ii) $\left[\xi^{\prime}(s) \xi^{\prime \prime}(s)\right]=-\sqrt{c(s)}$.

In the case $(i), \xi(s)$ has the following form

$$
\xi(s)=h+e^{i \varphi} \int_{c}^{s} e^{i \int_{v_{0}}^{v} \sqrt{c(u)} d u} d v
$$

where $\varphi \in \mathbb{R}$ is arbitrary.

In the case (ii), $\xi(s)$ has the following form

$$
\xi(s)=h+e^{i \varphi} \int_{c}^{s} e^{-i \int_{v_{0}}^{v} \sqrt{c(u)} d u} d v,
$$

where $\varphi \in \mathbb{R}$ is arbitrary and $v_{0} \in L_{\Phi}$.

Conversely, every path $\xi(s)$ of the form (7.3) or the form (7.4) is an invariant parametrization of a non-degenerate curve in $E_{2}$.

Proof. In the case $(i)$, we have $\left[\xi^{\prime}(s) \xi^{\prime \prime}(s)\right]=\sqrt{c(s)}$. Since $\left\langle\xi^{\prime}(s), \xi^{\prime}(s)>=a(s)=1\right.$ for all $s \in L_{\Phi}, \xi(s)$ has the form (7.3) by Theorem 7.1. In the case $(i i)$, we have $\left[\xi^{\prime}(s) \xi^{\prime \prime}(s)\right]=-\sqrt{c(s)}$. Similarly, by Theorem $7.1, \xi(s)$ has the form (7.4).

Theorem 7.5. . Let $\Phi$ be a non-degenerate curve in $E_{2}, \xi(s) \in P_{\Phi}$ and $d(s)$ be a real continuous function on $L_{\Phi}$ such that $d(s)>0$ for all $s \in L_{\Phi}$. Let $c \in L_{\Phi}, h \in E_{2}$ be given. Assume that $\xi(s) \in P_{\Phi}$ such that $\xi(c)=h$ and $\kappa(\xi(s))=d(s)$. Then, the following cases exist:

(i) $\left[\xi^{\prime}(s) \xi^{\prime \prime}(s)\right]=d(s)$,

(ii) $\left[\xi^{\prime}(s) \xi^{\prime \prime}(s)\right]=-d(s)$.

In the case $(i), \xi(s)$ has the following form

$$
\xi(s)=h+e^{i \varphi} \int_{c}^{s} e^{i \int_{v_{0}}^{v} d(u) d u} d v,
$$

where $\varphi \in \mathbb{R}$ is arbitrary.

In the case (ii), $\xi(s)$ has the following form

$$
\xi(s)=h+e^{i \varphi} \int_{c}^{s} e^{-i \int_{v_{0}}^{v} d(u) d u} d v
$$

where $\varphi \in \mathbb{R}$ is arbitrary and $v_{0} \in L_{\Phi}$.

Conversely, every path $\xi(s)$ of the form (7.5) or the form (7.6) is an invariant parametrization of a non-degenerate curve in $E_{2}$

Proof. Since $\xi(s)$ is an invariant parametrization, we have $\left\langle\xi^{\prime}(s), \xi^{\prime}(s)>=1, \forall s \in L_{\Phi}\right.$. Using $\left\langle\xi^{\prime}(s), \xi^{\prime}(s)>=\right.$ $1, \forall s \in L_{\Phi}$ and the equality $\kappa(\xi)=\frac{\left|\left[\xi^{\prime}(s) \xi^{\prime \prime}(s)\right]\right|}{\left\langle\xi^{\prime}(s), \xi^{\prime}(s)\right\rangle^{\frac{3}{2}}}=d(s)$, we obtain $\left|\left[\xi^{\prime}(s) \xi^{\prime \prime}(s)\right]\right|=d(s)$. This equality implies $\left[\xi^{\prime}(s) \xi^{\prime \prime}(s)\right]^{2}=d^{2}(s)$. Hence we obtain the equality $c(s)=d^{2}(s)$ for the function $c(s)$ in Theorem 7.4. Then, by Theorem $7.4, \xi(s)$ has the form (7.5) or the form (7.6).

Theorem 7.6. Let $\Phi$ be a non-degenerate curve in $E_{2}, \xi(s) \in P_{\Phi}$ and $d(s)$ be a real continuous function on $L_{\Phi}$ such that $d(s)>0$ for all $s \in L_{\Phi}$. Let $c \in L_{\Phi}, h \in E_{2}$ be given. Assume that $\xi(s) \in P_{\Phi}$ such that $\xi(c)=h$ and $<\xi^{\prime \prime}(s), \xi^{\prime \prime}(s)>=d(s)$. Then the following cases exist:

(i) $\left[\xi^{\prime}(s) \xi^{\prime \prime}(s)\right]=\sqrt{d(s)}$, 
(ii) $\left[\xi^{\prime}(s) \xi^{\prime \prime}(s)\right]=-\sqrt{d(s)}$.

In the case $(i), \xi(s)$ has the following form

$$
x(s)=h+e^{i \varphi} \int_{c}^{s} e^{i \int_{v_{0}}^{v} \sqrt{d(u)} d u} d v,
$$

where $\varphi \in \mathbb{R}$ is arbitrary and $v_{0} \in L_{\Phi}$.

In the case (ii), $\xi(s)$ has the following form

$$
\xi(s)=h+e^{i \varphi} \int_{c}^{s} e^{-i \int_{v_{0}}^{v} \sqrt{d(u)} d u} d v
$$

where $\varphi \in \mathbb{R}$ is arbitrary and $v_{0} \in L_{\Phi}$.

Conversely, every path $\xi(s)$ of the form (7.7) or the form (7.8) is an invariant parametrization of a non-degenerate curve in $E_{2}$.

Proof. The equality $<\xi^{\prime}(s), \xi^{\prime}(s)>=1, \forall s \in L_{\Phi}$, implies $<\xi^{\prime}(s), \xi^{\prime \prime}(s)>=0 \forall s \in L_{\Phi}$. Equalities $\left[\xi^{\prime}(s) \xi^{\prime \prime}(s)\right]^{2}=<$ $\xi^{\prime}(s), \xi^{\prime}(s)><\xi^{\prime \prime}(s), \xi^{\prime \prime}(s)>-<\xi^{\prime}(s), \xi^{\prime \prime}(s)>^{2},<\xi^{\prime}(s), \xi^{\prime}(s)>=1,<\xi^{\prime}(s), \xi^{\prime \prime}(s)>=0$ and $\left\langle\xi^{\prime \prime}(s), \xi^{\prime \prime}(s)>=\right.$ $d(s)$ imply the equality $\left[\xi^{\prime}(s) \xi^{\prime \prime}(s)\right]^{2}=d(s)$. Hence we obtain the equality $c(s)=d(s)$ for the function $c(s)$ in Theorem 7.4. Then, by Theorem 7.4, $\xi(s)$ has the form (7.7) or the form (7.8). By Theorem 7.4, the form (7.7) or the form (7.8) is an invariant parametrization of a non-degenerate curve in $E_{2}$.

\section{Conclusion}

Problems in computer vision and pattern recognition can be reduced to equivalence problem for paths and curves in $E_{2}$ or $E_{3}$ with respect to the actions of the Euclidean transformation groups. A solution of problems in this area can be given by finding evident form of the Euclidean transformations. (see $[13,14])$. In the present paper, by developing used method in the previous paper [15], for the group of Euclidean transformations in $E_{2}$, equivalence problems, existence and rigidity theorems for regular, completely regular and non-degenerate paths and curves are given. For given two paths and curves with the common differential $G$-invariants, we obtain, for the first time,evident forms of all Euclidean transformations that maps one of the paths and curves to the other.

\section{Acknowledgements}

The authors is very grateful to the reviewer(s) for helpful comments and valuable suggestions.

\section{References}

[1] Aminov, Yu.: Differential Geometry and Topology of Curves. CRC Press, New York, 2000.

[2] Aripov, R. G., Khadjiev (Khadzhiev) D.: The complete system of global differential and integral invariants of a curve in Euclidean geometry. Russian Mathematics (Iz VUZ), 51, No. 7, 1-14 (2007).

[3] Berger, M.: Geometry I. Springer-Verlag, Berlin Heidelberg, 1987.

[4] Gibson, C. G.: Elementary Geometry of Differentiable Curves. An Undergraduate Introduction, Cambridge University Press, 2001.

[5] Gray,A., Abbena, E. and Salamon,S.: Modern Differential Geometry of Curves and surfaces with Mathematica. Third edition. Studies in Advanced Mathematics. Chapman and Hall/CRC, Boca Raton, FL, 2006

[6] Guggenheimer, H. W.: Differential Geometry. Dower Publicatiýns, INC., New York, 1977.

[7] Khadjiev, D.: Application of the Invariant Theory to the Differential Geometry of Curves. "Fan" Publisher, Tashkent, 1988. [in Russian].

[8] Khadjiev, D.: Complete systems of differential invariants of vector fields in a Euclidean space. Turkish J. Math. 34, 543-560 (2010).

[9] Khadjiev, D.: On invariants of immersions of an n-dimensional manifold in an n-dimensional pseudo-euclidean space. Journal of Nonlinear Mathematical Physics, 17, Supp 01, 49-70 (2010).

[10] Khadjiev D., Pekşen Ö.: The complete system of global differential and integral invariants of equiaffine curves. Diff. Geom. And Appl. 20, 168-175 (2004).

[11] Khadjiev, D., Ören, İ., Pekşen, Ö.: Generating systems of differential invariants and the theorem on existence for curves in the pseudo-Euclidean geometry. Turkish J. Math. 37 , 80-94 (2013).

[12] Khadjiev, D., Ören, I., Pekşen, Ö.: Global invariants of path and curves for the group of all linear similarities in the two-dimensional Euclidean space. Int.J. Geo.Modern Phys,15(6), 1-28 (2018).

[13] Mundy, J.L., Zisserman, A.: Geometric Invariance in Computer Vision, Artificial Intelligence. MIT Press, Cambridge, 1992. 
[14] Mundy, J.L., Zisserman, A., Forsyth, D.: Application of Invariance in Computer Vision. Lecture Notes in Computer Science, Springer, Berlin, 1992.

[15] Khadjiev, D., Ören, İ.: Global invariants of paths and curves for the group of orthogonal transformations in the two-dimensional Euclidean space. An. St. Univ. Ovidius Constanta, 27(2), 37-65 (2019).

[16] Montel, S., Ros, A.: Curves and Surfaces. American Mathematical Society, 2005.

[17] Pekşen, Ö., Khadjiev, D.: On invariants of null curves in the pseudo-Euclidean geometry. Diff. Geom. and Appl. 29, 183-187 (2011).

[18] Pekşen, Ö., Khadjiev, D., Ören, I.: Invariant parametrizations and complete systems of global invariants of curves in the pseudo-Euclidean geometry. Turkish J. Math. 36, 147-160 (2012).

[19] Spivak, M.: Comprehensive Introduction to Differential Geometry. Vol. 2 Publish Or Perish, INC., Houston, Texas, 1999.

[20] Toponogov, V. A.: Differential Geometry of Curves and Surfaces. Birkhauser, Boston, 2006.

\section{Affiliations}

İDRIS ÖREN

ADDRESS: Karadeniz Technical University, Dept. of Mathematics, 61080, Trabzon, Turkey

E-MAIL: oren@ktu.edu.tr

ORCID ID: 0000-0003-2716-3945

DJAVVAT KHADJIEV

AdDress: National University of Uzbekistan, Institute of Mathematics, Tashkent,Uzbekistan

E-MAIL: khadjiev@gmail.com

ORCID ID: 0000-0001-7056-5662 